\title{
Two- and three-dimensional models for analysis of optical absorption in tungsten disulphide single crystals
}

\author{
DHAIRYA A DHOLAKIA, G K SOLANKI*, S G PATEL and M K AGARWAL \\ Department of Physics, Sardar Patel University, Vallabh Vidyanagar 388 120, India
}

MS received 4 September 2000; revised 20 February 2001

\begin{abstract}
The optical energy gaps of $\mathrm{WS}_{2}$ single crystal were determined from the analysis of the absorption spectrum near the fundamental absorption edge at room temperature using light parallel to $c$-axis incident normally on the basal plane. On the basis of two- and three-dimensional models it was found that both direct and indirect band transitions took place in $\mathrm{WS}_{2}$ and the indirect transition was of the allowed type. The optical energy gaps corresponding to both transitions were determined and the phonon energies associated with the indirect transitions estimated. The implications of the results have been discussed.
\end{abstract}

Keywords. Optical band gap; two- and three-dimensional; optical absorption.

\section{Introduction}

Tungsten disulphide crystallizes with a hexagonal $2 \mathrm{H}$ $\mathrm{MoS}_{2}$ structure (C7 type). The tungsten atoms are in trigonal prismatic coordination between two superimposable sheets of hexagonally packed sulphur atoms. No strong bond exists between the layers, however, only long range van der Waals forces hold atomic sandwiches together. This gives the crystals their characteristic platy habit, with extended growth and pronounced cleavage perpendicular to $c$-axis. The stacking sequence is ABA, $\mathrm{BAB}$ with the space group $D_{6 h}^{4}\left(\mathrm{~Pb}_{3} / \mathrm{mmc}\right)$ having lattice parameters $a=3.78 \pm 0.022 \AA$ and $c=12 \cdot 362 \pm 0.003 \AA$.

The structure of layered tungsten disulphide $\left(\mathrm{WS}_{2}\right)$ can be considered to be the result of stacking of twodimensional slabs [S W S] built from two anionic (S) planes in which a cationic (W) one is enclosed. In each slab/layer, bondings are strong, between slabs they are weak and belong to the van der Waals model. The twodimensional sandwiches are stacked on top of each other to form a three-dimensional solid. If the bonding between the sandwiches/layers is neglected then it leads to a twodimensional model in which each layer is effectively independent from neighbouring layers. However, if the bonding between the layers is not neglected then it leads to a three-dimensional model. A question always persists whether $\mathrm{WS}_{2}$ (belonging to the $\mathrm{MoS}_{2}$ family) are truly two-dimensional systems or are closer to three-dimensional ones. Any physical property measurement which helps in resolving this issue should therefore be welcome. Elkorashy (1986a) successfully used two- and three-

\footnotetext{
*Author for correspondence
}

dimensional models for the analysis of optical absorption in layered tin sulphide single crystals. He observed that the two-dimensional model gave the same value of the optical energy gap for $\mathrm{SnS}$ as that obtained from threedimensional model with the difference between the two models being less than $2 \%$. Although a great deal of work on the optical band gap measurements (Frindt 1963; Goldberg et al 1975; Kam and Parkinson 1982; Baglio et al 1982, 1983; Oritz 1995; Li et al 1996) has been carried out on $\mathrm{WS}_{2}$, there is no detailed analysis of the absorption spectra obtained from the single crystals of $\mathrm{WS}_{2}$ on the basis of two- and three-dimensional models. We have therefore carried out this study and the results and their implications thus obtained are presented here.

\section{Experimental}

All the samples for the present work were grown by a direct vapour transport technique (Al-Hilli and Evans 1972). As grown crystals exhibit $p$-type conductivity with a hole concentration at room temperature of $10^{16} \mathrm{~cm}^{-3}$. Since the crystals grew in the form of thin platelets, 'as grown' samples could be directly used for the absorption measurements. The surfaces of these samples were mirror like. The $a$ - and $b$-axes were confined in the plane of cleavage i.e. in the basal plane. The optical absorption data were obtained by means of a DK 2A Spectrophotometer. Measurements were performed at room temperature with the incident light beam normal to the basal plane i.e. along the $c$-axis of the grown flakes. Measurements along the $c$-axis were not performed because the crystal structure did not permit cutting and polishing. 


\section{Results}

Figure 1 shows the absorption spectrum taken from a $\mathrm{WS}_{2}$ single crystal in the form of a thin platelet over the spectral range $700-1450 \mathrm{~nm}$. A careful study of this spectrum reveals the presence of an absorption edge in the spectral range $700-850 \mathrm{~nm}$. In order to analyse the results in the vicinity of the absorption edge on the basis of twoas well as three-dimensional models, values of absorption coefficient ' $\alpha$ ' were determined at every step of $2.5 \mathrm{~nm}$. The interpretation of experimental results, viz. the dependence of absorption coefficient ' $\alpha$ ' in terms of the direct and indirect transitions is most often performed with the help of the formulae derived for three-dimensional (3D) crystals. Their simplest form is as follows (Pankov and Jaques 1975)

$$
\alpha h v=A\left(h v-E_{\mathrm{g}}\right)^{r},
$$

for direct transitions and

$$
\alpha h v=\sum_{j} B_{j}\left(h v-E_{\mathrm{g}}^{\prime} \pm E_{\mathrm{pj}}\right)^{r},
$$

for indirect transitions.

Here, $\alpha$ is the absorption coefficient, $h v$ the energy of the incident photon, $E_{\mathrm{g}}$ the energy for the direct transition, $E_{\mathrm{g}}^{\prime}$ the energy for the indirect transition, $E_{\mathrm{pj}}$ the energies of the phonons assisting at indirect transitions, $A$ and $B$ are parameters depending in a more complicated way on temperature, photon energy and phonon energies $E_{\mathrm{p}}$. However, for the analysis of the experimental results obtained at constant temperature, (1) and (2) are sufficient and they are most often used while interpreting results on absorption spectra obtained from semiconducting materials. The exponent $r$ in above equations depends upon whether the transition is symmetry allowed or not and the constants $A$ and $B$ will assume different values for the allowed and forbidden transitions. For indirect transitions, the detailed form of (2) (Vlachs et al 1976; Elkorashy $1986 \mathrm{~b}, \mathrm{c})$ is given as

$$
\begin{aligned}
\alpha_{i}= & \sum_{i=1}^{2}\left\{\frac{B_{a i}}{E}\left(\frac{1}{e^{\theta_{i / T}}-1}\right)\left(E-E_{\mathrm{g}}^{\prime}+k \theta_{i}\right)^{r}\right. \\
& \left.+\frac{B_{e i}}{E}\left(\frac{1}{1-e^{-\theta_{i / T}}}\right)\left(E-E_{\mathrm{g}}^{\prime}-k \theta_{i}\right)^{r}\right\},
\end{aligned}
$$

where $B_{a i}$ and $B_{e i}$ are coefficients associated with absorption and emission of the $i$ th phonon, $E$ the photon energy, $E_{\mathrm{g}}^{\prime}$ the indirect energy gap and $\theta_{i}$ is a phonon equivalent temperature defined by the equation

$$
E_{p i}=k \theta_{i}
$$

( $E_{p i}$ being the $i$ th phonon energy). The use of (1)-(3) for analysing the absorption spectrum is valid for semi- conductors having a three-dimensional structure, but for anisotropic layered materials like $\mathrm{WS}_{2}$, one has to assume a two-dimensional form of the density of states as discussed by Fivaz (1967) and Brebner (1964). In these cases the density of states is a constant independent of the energy and the expressions showing the dependence of $\alpha$ in terms of direct and indirect transitions get modified (Elkorashy 1988) as

$$
\alpha=A^{\prime}\left(h v-E_{\mathrm{g}}\right)^{r}
$$

(Goldberg et al 1975).

$$
\begin{aligned}
\alpha_{i}= & \sum_{i=1}^{2} B_{a i}^{\prime}\left\{\left(\frac{1}{e^{\theta_{i / T}}-1}\right)\left(E-E_{\mathrm{g}}^{\prime}+k \theta_{i}\right)^{r}\right. \\
& \left.+B_{e i}^{\prime}\left(\frac{1}{1-e^{-\theta_{i / T}}}\right)\left(E-E_{\mathrm{g}}^{\prime}-k \theta_{i}\right)^{r}\right\},
\end{aligned}
$$

(Elkorashy 1988).

The symbols in (4) and (5) have the same meaning as explained earlier in (1)-(3). Again, the exponent $r$ depends on the dimensionality of the bands and whether the

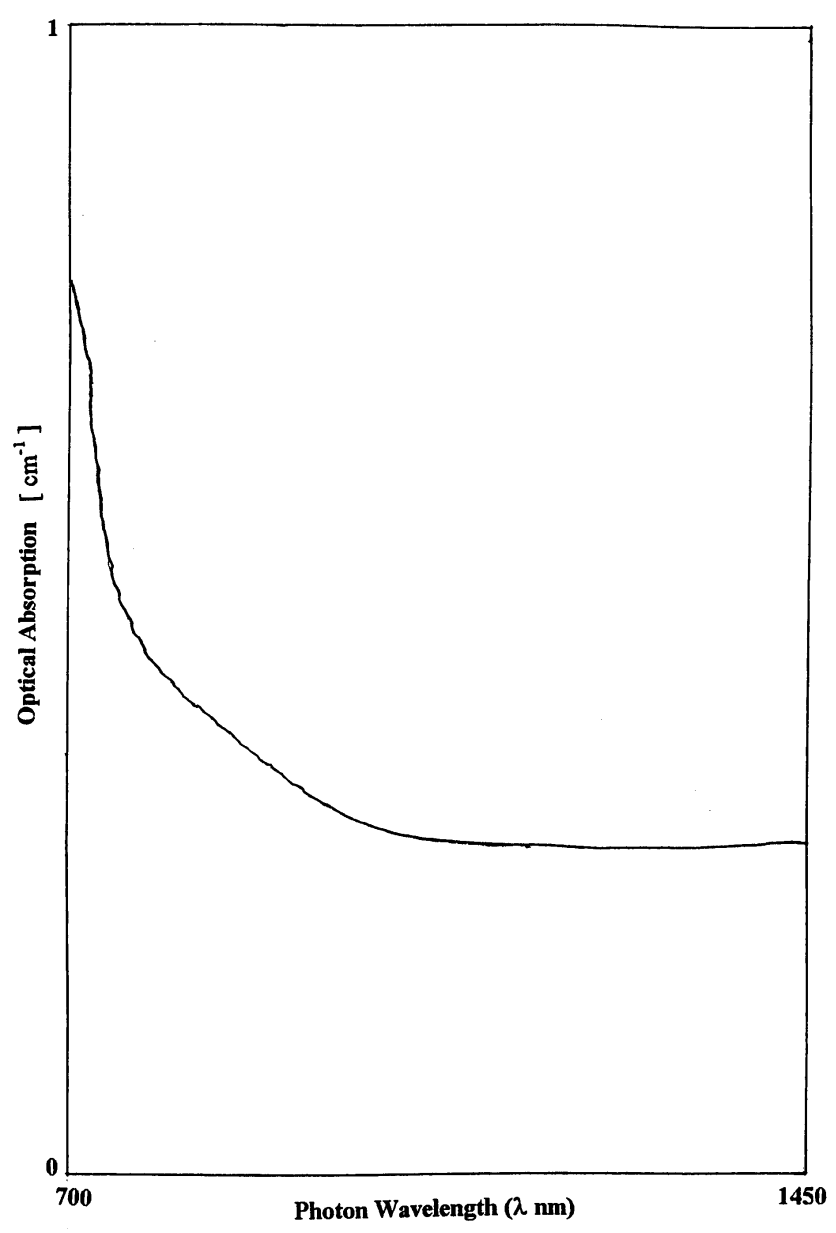

Figure 1. Absorption spectrum from a $\mathrm{WS}_{2}$ single crystal. 
transitions are symmetry allowed or forbidden. Once again the coefficients $A^{\prime}, B_{a i}^{\prime}$ and $B_{e i}^{\prime}$ will be different for symmetry allowed and forbidden transitions. Possible values of $r$ are given in table 1 (Lee et al 1969; Goldberg et al 1975; Kam et al 1984).

By plotting graphs of $(\alpha h v)^{1 / r}$ against $h v$ for various values of $r$ given in table 1 , it is possible to determine which of the conditions given in the table dominate. Extrapolations of these plots to zero absorption will give the appropriate value of the energy gaps of $\mathrm{WS}_{2}$.

Accordingly, figure 2 shows the spectral variation of $(\alpha h v)^{1 / 2}$ vs $h v$. Since the curve indicates discontinuous straight line, it is quite plausible that it represents indirect interband transitions involving the emission or absorption of phonons. In order to make an accurate determination of the points of discontinuities, we have followed the method adopted by Koshkin et al (1970) and Elkorashy (1986c). From the graphical differentiation of the data presented in figure 2 , the dependence of the derivatives $\partial(\alpha h v)^{1 / 2} / \partial h v$ on $h v$ has been shown in figure 3. It can be clearly seen from this figure that the derivatives are step functions of energy with four steps well defined in the range.

$$
\begin{aligned}
& E_{1}<E<E_{2}, \\
& E_{2}<E<E_{3}, \\
& E_{3}<E<E_{4}, \\
& E_{4}<E .
\end{aligned}
$$

The values of $E_{1}, E_{2}, E_{3}$ and $E_{4}$ indicate the points of discontinuities in the plot of $\partial(\alpha h v)^{1 / 2} / \partial h v$ vs $h v$. The indirect energy gaps obtained from these values of $E_{1}, E_{2}$, $E_{3}$ and $E_{4}$ are given by

$$
E_{\mathrm{g}}^{\prime}=\frac{E_{1}+E_{4}}{2}=\frac{E_{2}+E_{3}}{2},
$$

and the phonon energies are given by

$$
E_{\mathrm{p} 1}=\frac{E_{4}-E_{1}}{2} \text { and } E_{\mathrm{p} 2}=\frac{E_{3}-E_{2}}{2} .
$$

The values of indirect band gap $E_{\mathrm{g}}^{\prime}$ and phonon energies thus obtained are given in table 2 . The value of $E_{\mathrm{g}}^{\prime}$ can also be obtained from the intersection of the linear portion of the graph in figure 2 with the energy axis for zero absorption. This value closely matches with the value obtained from (6).

Table 1. Values of exponent ' $r$ ' for different types of band gap transitions.

\begin{tabular}{lccccc}
\hline & \multicolumn{2}{c}{ Direct } & & \multicolumn{2}{c}{ Indirect } \\
\cline { 2 - 3 } \cline { 5 - 6 } Type of transition & Two-dimensional & Three-dimensional & & Two-dimensional & Three-dimensional \\
\hline Allowed & 0 & $1 / 2$ & 1 & 2 \\
Forbidden & $\begin{array}{c}\text { (Step function) } \\
1\end{array}$ & $3 / 2$ & 2 & 3 \\
\hline
\end{tabular}

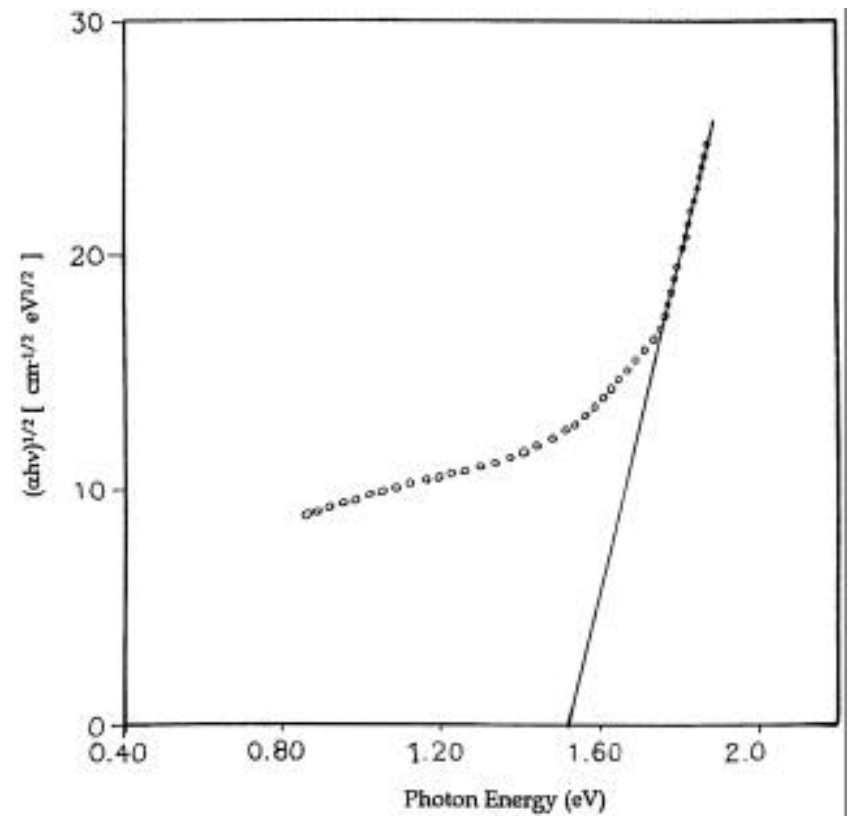

Figure 2. The spectral variation of the quantity $(\alpha h v)^{1 / 2}$.

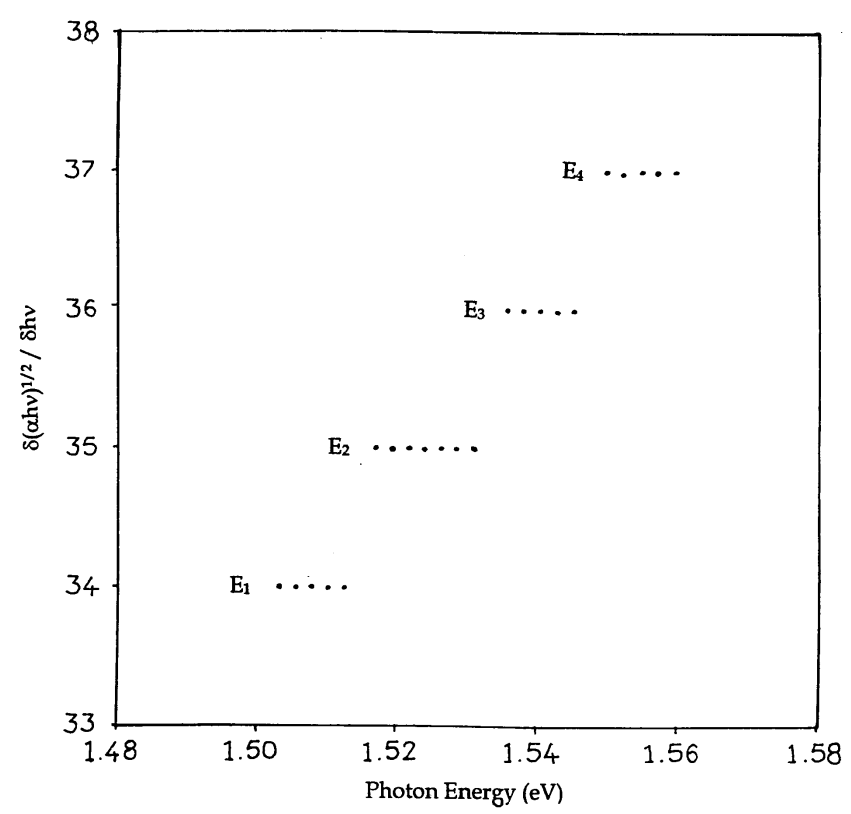

Figure 3. The spectral variation of the derivative $\delta(\alpha h v)^{1 / 2} /$ $\delta h v$ obtained by graphical differentiation of the data presented in figure 2. 
Now a graph of $(\alpha h v)^{1 / 3}$ vs $h v$ is shown in figure 4 . It is seen that the nature of this graph widely differs from that shown in figure 2. A graphical differentiation of the data presented in this figure and the intersection of the straight line portion of the graph with the energy axis for zero absorption will give widely different values. It is, therefore, conjectured that the indirect transition represented by the absorption curve is an indirect allowed type.

In order to analyse the data from the absorption curve on the basis of two-dimensional model, variation of $\alpha^{1 / 2}$ vs $h v$ was studied. A graph showing the spectral variation of $\alpha^{1 / 2}$ is shown in figure 5. A graphical differentiation of the data presented in this figure is shown in figure 6 . The values of indirect band gap $E_{\mathrm{g}}^{\prime}$ obtained from the extrapolation of the linear portion of the curve in figure 5 and

Table 2. Indirect (allowed) band gap and phonon energies for $\mathrm{WS}_{2}$.

\begin{tabular}{lccl}
\hline Parameter & $\begin{array}{c}\text { Three- } \\
\text { dimensional } \\
\text { model }\end{array}$ & $\begin{array}{c}\text { Two- } \\
\text { dimensional } \\
\text { model }\end{array}$ & $\begin{array}{l}\text { Values of } E_{\mathrm{g}}^{\prime} \\
\text { obtained by } \\
\text { earlier workers }\end{array}$ \\
\hline$E_{1}(\mathrm{eV})$ & 1.50 & 1.47 & $\begin{array}{l}1.33 \text { Baglio } \text { et al } \\
(1982)\end{array}$ \\
$E_{2}(\mathrm{eV})$ & 1.51 & 1.49 & $\begin{array}{l}1.35 \text { Kam and } \\
\text { Parkinson }(1982)\end{array}$ \\
$E_{3}(\mathrm{eV})$ & 1.53 & 1.52 & \\
$E_{4}(\mathrm{eV})$ & 1.50 & 1.54 & \\
$E_{\mathrm{g}}^{\prime}(\mathrm{C})(\mathrm{eV})$ & 1.52 & 1.50 & \\
$E_{\mathrm{g}}^{\prime}(\mathrm{E})(\mathrm{eV})$ & 1.52 & 1.50 & \\
$E_{\mathrm{p} 1}(\mathrm{MeV})$ & 23.41 & 36.57 & \\
$E_{\mathrm{p} 2}(\mathrm{MeV})$ & 9.36 & 11.45 & \\
\hline
\end{tabular}

$E_{\mathrm{g}}^{\prime}(\mathrm{C})$, indirect band gap from calculation; $E_{\mathrm{g}}^{\prime}(\mathrm{E})$, indirect band gap from extrapolation.

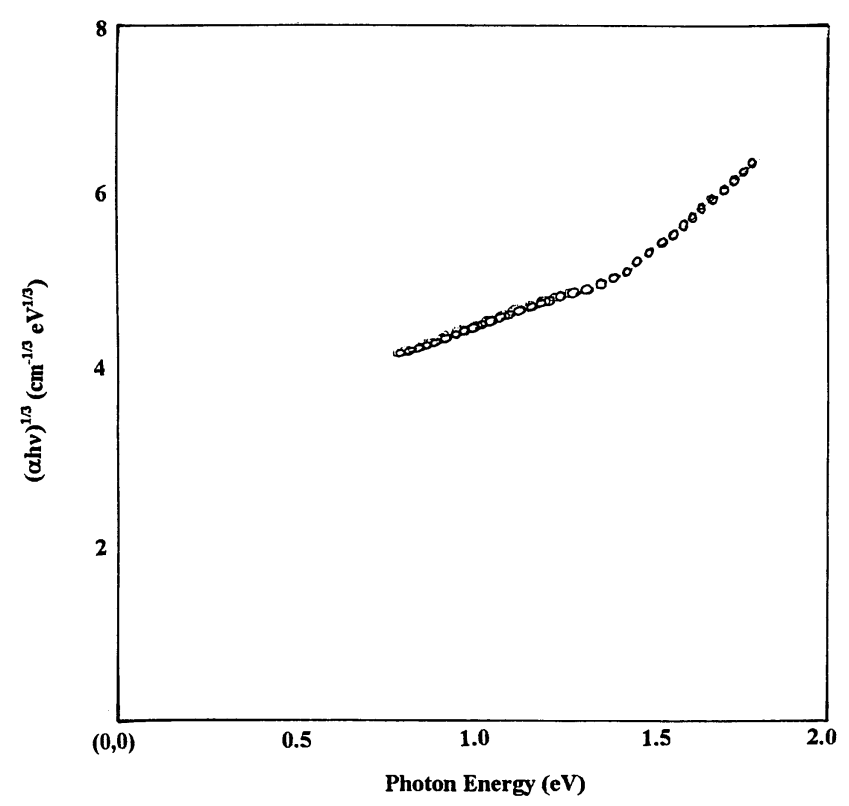

Figure 4. The spectral variation of the quantity $(\alpha h v)^{1 / 3}$. that obtained from the graphical differentiation of the data in figure 6 are shown in table 2. The values of phonon energies obtained from figure 6 are also given in this table. Taking the values of $E_{\mathrm{g}}^{\prime}$ and $E_{\mathrm{p} 1}$ at room temperature from table 2 , the constants $B_{\mathrm{a} 1}, B_{\mathrm{a} 2}, B_{\mathrm{e} 1}, B_{\mathrm{e} 2}, \theta_{1}$ and $\theta_{2}$ appearing in (3) and similar constants $B_{\mathrm{a} 1}^{\prime}, B_{\mathrm{a} 2}^{\prime}, B_{\mathrm{e} 1}^{\prime}, B_{\mathrm{e} 2}^{\prime}, \theta_{1}$ and $\theta_{2}$ appearing in (5) have been determined with the help of (3) and (5) taking $r=3$ for three-dimensional

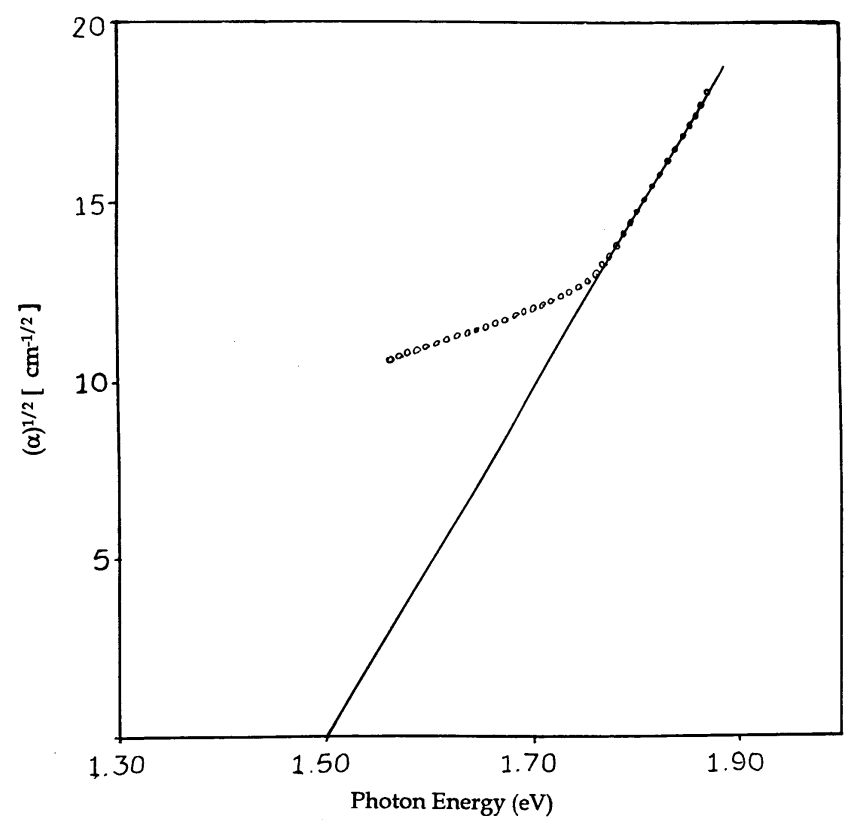

Figure 5. The spectral variation of the quantity $(\alpha)^{1 / 2}$.

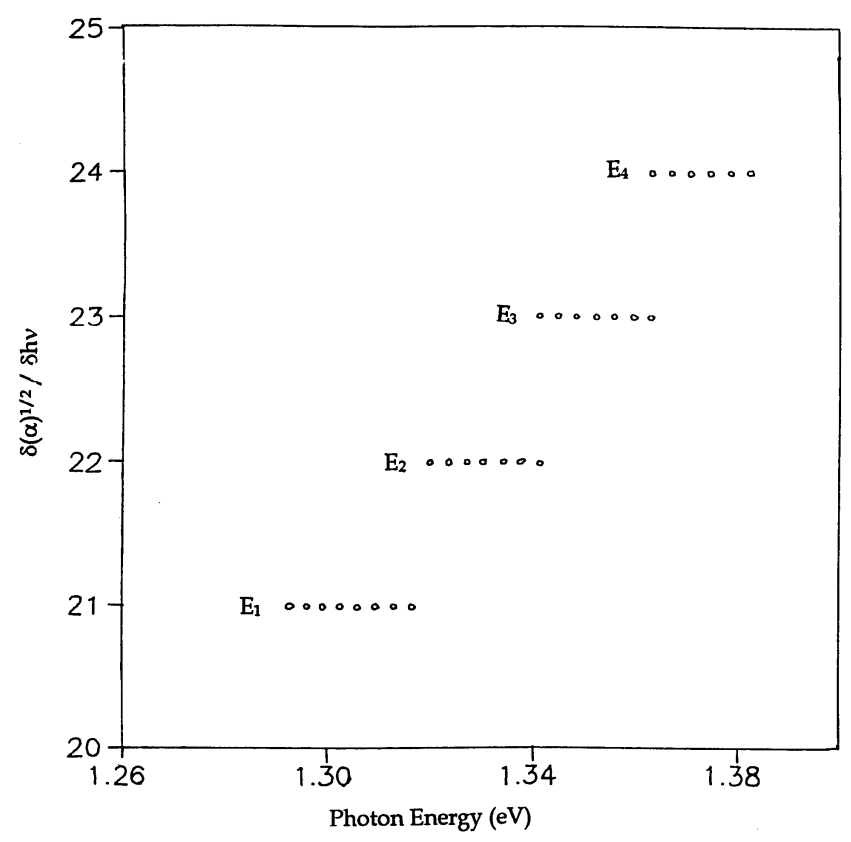

Figure 6. The spectral variation of the derivative $\delta(\alpha)^{1 / 2} / \delta h v$ obtained by graphical differentiation of the data presented in figure 5 . 
Table 3. The constants of (3) and (5) for $\mathrm{WS}_{2}$.

\begin{tabular}{lclc}
\hline Constants in (3) & Three-dimensional model & Constants in (5) & Two-dimensional model \\
\hline$B_{\mathrm{e} 1}\left(\mathrm{~cm}^{-1} \mathrm{eV}^{-2}\right)$ & $1246830 \cdot 5$ & $B_{\mathrm{e} 1}^{\prime}\left(\mathrm{cm}^{-1} \mathrm{eV}^{-2}\right)$ & $45410 \cdot 9$ \\
$B_{\mathrm{a} 1}\left(\mathrm{~cm}^{-1} \mathrm{eV}^{-2}\right)$ & $7346683 \cdot 0$ & $B_{\mathrm{a} 1}^{\prime}\left(\mathrm{cm}^{-1} \mathrm{eV}^{-2}\right)$ & $511863 \cdot 1$ \\
$B_{\mathrm{e} 2}\left(\mathrm{~cm}^{-1} \mathrm{eV}^{-2}\right)$ & $3132951 \cdot 3$ & $B_{\mathrm{e} 2}^{\prime}\left(\mathrm{cm}^{-1} \mathrm{eV}^{-2}\right)$ & $490082 \cdot 1$ \\
$B_{\mathrm{a} 2}\left(\mathrm{~cm}^{-1} \mathrm{eV}^{-2}\right)$ & $6328467 \cdot 7$ & $B_{\mathrm{a} 2}^{\prime}\left(\mathrm{cm}^{-1} \mathrm{eV}^{-2}\right)$ & $1025043 \cdot 6$ \\
$\theta_{1}(\mathrm{~K})$ & $271 \cdot 46$ & $\theta_{1}(\mathrm{~K})$ & $424 \cdot 02$ \\
$\theta_{2}(\mathrm{~K})$ & $108 \cdot 57$ & $\theta_{2}(\mathrm{~K})$ & $132 \cdot 84$ \\
\hline
\end{tabular}

Table 4. Direct energy gap for $\mathrm{WS}_{2}$.

\begin{tabular}{lcc}
\hline Model & Values of $E_{\mathrm{g}}(\mathrm{eV})$ & Values of $E_{\mathrm{g}}$ obtained by earlier workers \\
\hline Three-dimensional & 1.80 & 1.79 Kam and Parkinson $(1982)$ \\
Two-dimensional & 1.78 & 1.78 Baglio et al $(1982)$ \\
& & 1.80 Baglio et al $(1983)$ \\
& & 1.77 Vashi $(1985)$ \\
\hline
\end{tabular}

model and $r=2$ for two-dimensional model. All these values are presented in table 3 .

For the determination of the direct band gap $E_{\mathrm{g}}$, the spectral variation of $(\alpha h v)^{2}$ vs $h v$ as shown in figure 7 was studied. The value of $E_{\mathrm{g}}$ obtained from the intersection of the straight line portion of the curve on the $h \mathrm{v}$ axis for zero absorption is shown in table 4. In order to obtain the value of $E_{\mathrm{g}}$ on the basis of two-dimensional model the spectral variation of $\alpha^{2}$ was studied (figure 8). Here also the value of $E_{\mathrm{g}}$ obtained from the extrapolation of the straight line portion of the curve on energy axis for zero absorption was determined (table 4).

\section{Discussion}

From table 2, it is clearly evident that the indirect optical energy gap in $\mathrm{WS}_{2}$ is indirect allowed with absorption and emission of the phonons with energies 23.41 and $9.36 \mathrm{meV}$ respectively. The values of direct band gap for $\mathrm{WS}_{2}$ represented in table 4 clearly confirm that the transition is direct allowed. From tables 2 and 4, we come to the conclusion that the two-dimensional model gives nearly the same values of the optical energy gap for $\mathrm{WS}_{2}$ as those obtained from three-dimensional model with a difference between the two models being $\sim 2 \%$. Thus we see that both direct and indirect symmetry allowed transitions give a good account of the optical absorption edge in $\mathrm{WS}_{2}$. It may be seen from table 4 , that there is a good deal of agreement between the values of direct band gap obtained by us with the results of earlier researchers in this field. The same is not true for the values of the indirect band gap (table 2). This is because the values obtained by Kam and Parkinson (1982) and Baglio et al (1982) are based on the analysis of photocurrent and surface photovoltage spectra, whereas the detailed analysis obtained in the present work is based on the optical absorption spectra.

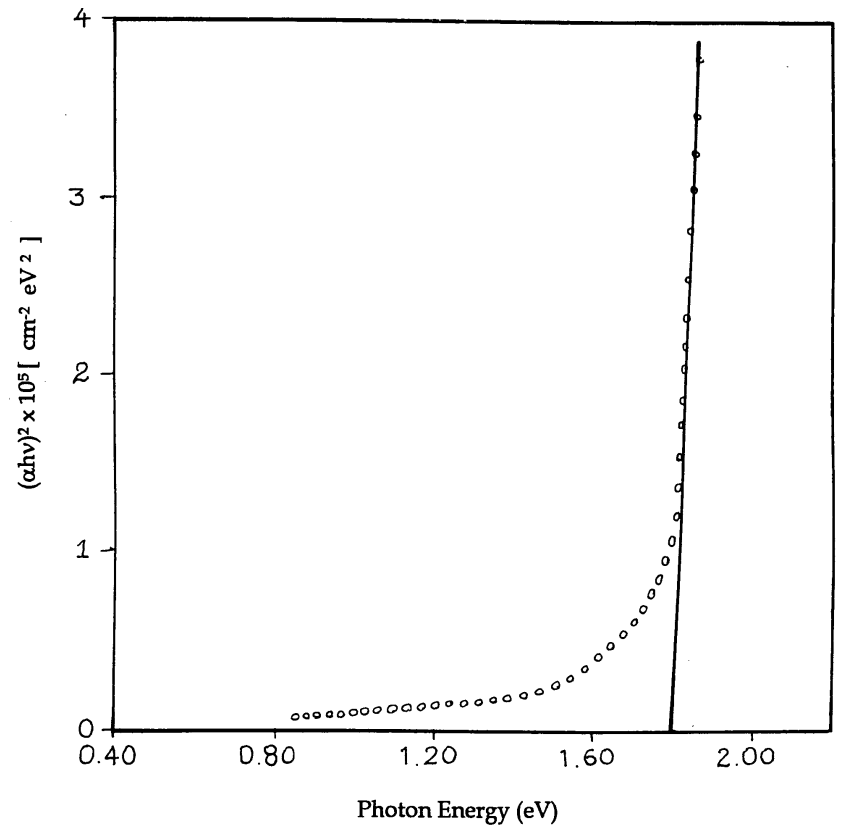

Figure 7. The spectral variation of the quantity $(\alpha h v)^{2}$.

The fact that two-dimensional model can also be used satisfactorily to describe the main optical properties of $\mathrm{WS}_{2}$ single crystals supports the high anisotropic nature of these compounds and two-dimensional behaviour seems likely for these compounds. The anisotropic behaviour of $\mathrm{WS}_{2}$ is also reflected in the room temperature resistivity measurements made along its basal plane and normal to the basal plane. The anisotropy ratio is defined as

$$
\gamma=\left(\rho_{\|} / \rho_{\perp}\right)
$$

where $\rho_{\|}$is the resistivity along the basal plane and $\rho_{\perp}$ the resistivity normal to the basal plane i.e. parallel to the $c$ axis. Value of $\gamma$ for $\mathrm{WS}_{2}$ comes out to be as high as 


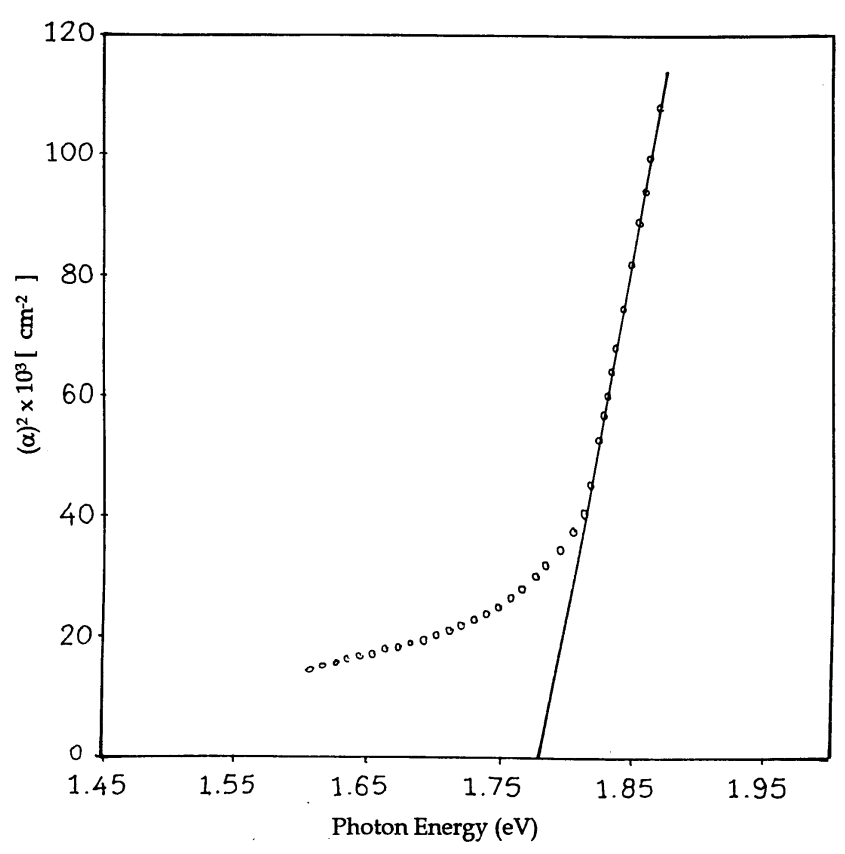

Figure 8. The spectral variation of the quantity $(\alpha)^{2}$.

$3.47 \times 10^{4}$. The possibility of accounting for the absorption data on the three-dimensional model clearly suggests that although $\mathrm{WS}_{2}$ compounds possess fair amount of anisotropy, they do possess some conductivity along the $c$-axis. The value of conductivity $\left[0 \cdot 13(\mu \Omega \mathrm{cm})^{-1}\right]$ obtained during measurements parallel to $c$-axis clearly confirm this conjecture. The transport of charge carriers perpendicular to the layers can be explained by assuming the existence of stacking faults in $\mathrm{WS}_{2}$, presence of such faults leads to a possibility of localization of electron states in a direction normal to the layers. This will give rise to conduction parallel to $c$-axis, thus making the crystals of $\mathrm{WS}_{2}$ three-dimensional. The presence of stacking faults has been recently shown in $\mathrm{WS}_{2}$ single crystals by Agarwal et al (1999).

\section{Conclusions}

The results described in this paper attempt to provide some explanation for the problem of dimensionality of the layer like compounds e.g. $\mathrm{WS}_{2}$. The possibility of analysing the absorption data from $\mathrm{WS}_{2}$ single crystals on the basis of two- and three-dimensional models clearly suggest us to conclude that, it is perhaps not appropriate to classify $\mathrm{WS}_{2}$ as a truely layer like (two-dimensional) compound but, rather, it should be considered as an intermediate case between layer like two-dimensional (2D) and three-dimensional (3D) or more appropriately as pseudo two-dimensional system.

\section{Acknowledgement}

Financial support from the University Grants Commission, New Delhi, in the form of a research project is gratefully acknowledged.

\section{References}

Agarwal M K, Patel Y and Vashi M N 1999 Indian J. Pure \& Appl. Phys. 37464

Al-Hilli A A and Evans B L 1972 J. Cryst. Growth 1593

Baglio J A et al 1982 J. Electrochem. Soc. 1291461

Baglio J A et al 1983 J. Am. Chem. Soc. 1052246

Brebner J C 1964 J. Phys. Chem. Solids 251427

Elkorashy A M 1986a Egypt J. Phys. 1739

Elkorashy A M 1986b Phys. Status Solidi (b) 135707

Elkorashy A M 1986c J. Phys. Chem. Solids 47497

Elkorashy A M 1988 Phys. Status Solidi (b) 146279

Fivaz R 1967 J. Phys. Chem. Solids 28839

Frindt R F 1963 J. Phys. Chem. Solids 241107

Goldberg A M, Beal A R, Levy F A and Davis E A 1975 Philos. Mag. 32367

Kam K K and Parkinson B A 1982 J. Phys. Chem. 86463

Kam K K, Chang C L and Lynch D W 1984 J. Phys. C: Solid State Phys. 174031

Koshkin V M, Karas V R and Gal'chinetskii P 1970 Sov. Phys. Semicond. 31186

Lee P A, Said G, Davis R and Lim T H 1969 J. Phys. Chem. Solids 302719

Li S J, Bernede J C, Pouzet J and Jamali M 1996 J. Phys. Condensed Matter 82291

Oritz Y S, Torres G I, Diaz A and Cabrera C R $1995 \mathrm{~J}$. Electrochem. Soc. 1422770

Pankov J I 1975 Optical processes in semiconductors (New York: Dover Publ. Inc.) p. 1

Vashi M N 1985 Growth and characterization of tungsten sulphoselenide single crystals with their use in photoelectrochemical solar cells, Ph.D. Thesis, Sardar Patel University, Vallabh Vidyanagar

Vlachs S V, Lambros A P, Thanailakis A and Economou N A 1976 Phys. Status Solidi (b) 76727 